\begin{tabular}{|c|c||c||}
\hline JOURNAL OF EDUCATION, SCIENCE AND HEALTH - JESH & Revista de Educação, Ciência e Saúde \\
DOI: https://www.doi.org/10.52832/jesh.v2i1.92 & Home page: $\underline{w w w . j e s h j o u r n a l . c o m . b r}$ \\
\hline
\end{tabular}

\title{
UM ESTUDO DA FIBRILAÇÃO ATRIAL SOB UMA PERSPECTIVA EPIDEMIOLÓGICA ATRELADA AOS FATORES DE RISCO ENVOLVIDOS - UMA REVISÃO
}

\author{
A STUDY OF ATRIAL FIBRILLATION UNDER AN EPIDEMIOLOGICAL PERSPECTIVE LINKED TO THE RISK \\ FACTORS INVOLVED - A REVIEW
}

UN ESTUDIO DE LA FIBRILACIÓN AURICULAR BAJO UNA PERSPECTIVA EPIDEMIOLÓGICA VINCULADA A LOS FACTORES DE RIESGO INVOLUCRADOS - UMA REVISIÓN

\begin{abstract}
Bianca Caldeira Leite ${ }^{1 *}$ (D) Artur Moreno de Andrade Vasconcelos ${ }^{1}{ }^{(D}$; Cibele Lorena Fernandes Guerra1 ${ }^{1}$; Victória Sampaio Moreira ${ }^{1}{ }^{\mathbb{D}}$; Júlia Thaís Cruz ${ }^{1 \mathbb{D}}$; Ubiraídys de Andrade Isidório ${ }^{\mathbb{D}}$; Marta Lígia Vieira Melo $^{3}$ (D)

${ }^{1}$ Estudante de Medicina. Faculdade Santa Maria (FSM), Cajazeiras, Paraíba, Brasil; ${ }^{2}$ Mestre, Universidade Cruzeiro do Sul (UNICSUL). Professor da Faculdade Santa Maria (FSM), Cajazeiras, Paraíba, Brasil; ${ }^{3}$ Mestra, Universidade Católica de Santos (UNISANTOS). Orientadora/Professora da Faculdade Santa Maria (FSM), Cajazeiras, Paraíba, Brasil.

*Autor correspondente: bicleite04@gmail.com.
\end{abstract}

Recebido: 12/01/2022 | Aprovado: 04/02/2022 | Publicado: 19/02/2022

\begin{abstract}
Resumo: A fibrilação atrial é uma disfunção do ritmo cardíaco ocasionada por uma excitabilidade de alta frequência e desordenada dos átrios. A estrutura elétrica e as células que constituem as veias pulmonares são os provedores da reentrada e da atividade ectópica da fibrilação atrial. Desse modo, esse estudo busca apontar dados referentes à epidemiologia da fibrilação atrial e os fatores de riscos relacionados à tal patologia. Trata-se de uma revisão integrativa da literatura realizada por intermédio da seleção de artigos publicados em periódicos indexados nas bases de dados do SciELO e PubMed. Ao todo, somaram-se 329 artigos encontrados, destes, 20 literaturas foram selecionadas para compor esta revisão. A fibrilação atrial apresenta prevalência média de 33,5 milhões de pessoas em todo o globo. As taxas de prevalência mais elevadas de fibrilaç são encontradas nos países desenvolvidos, principalmente os da América do Norte. A relevância do sexo no desenvolvimento da doença é discutível, e parece ser semelhante, exceto em maiores de 75 anos, cujo risco será maior em mulheres devido ao aumento da longevidade destas. Os fatores de risco de fibrilação atrial podem gerar alterações atriais, as quais podem ser estruturais ou não, que predispõem pacientes a desenvolver fibrilação atrial. Alguns desses fatores de risco mais comuns incluem diabetes mellitus, alcoolismo, tabagismo e obesidade. A relevância diferença de sexo quanto a um fator predisponente à fibrilação atrial ainda é inconclusiva, mas comprovou-se que os fatores supracitados aumentam o risco de manifestação de fibrilação atrial.
\end{abstract}

Palavras-chave: Epidemiologia. Fatores de risco. Fibrilação Atrial.

Abstract: The atrial fibrillation is a dysfunction of the heart rhythm caused by high-frequency and disordered excitability of the atria. The electrical structure and cells that make up the pulmonary veins are the providers of reentry and ectopic activity in atrial fibrillation. Thus, this study seeks to point out data regarding the epidemiology of atrial fibrillation and the risk factors related to this pathology. This is an integrative literature review carried out through the selection of articles published in journals indexed in the SciELO and PubMed databases. In all, 329 articles were found, of which 20 literatures were selected to compose this review. Atrial fibrillation has an average prevalence of 33.5 million people across the globe. The highest prevalence rates of atrial fibrillation are found in developed countries, mainly in North America. The relevance of sex in the development of the disease is debatable, and it seems to be similar, except in people over 75 years of age, whose risk will be greater in women due to their increased longevity. Atrial fibrillation risk factors can generate atrial changes, which may or may not be structural, which predispose patients to develop atrial fibrillation. Some of these more common risk factors include diabetes mellitus, alcoholism, smoking, and obesity. The relevance of the sex difference as a predisposing factor to atrial fibrillation is still inconclusive, but the aforementioned factors have been shown to increase the risk of manifestation of atrial fibrillation.

Keywords: Epidemiology. Risk factors. Atrial fibrillation.

Resumen: La fibrilación auricular es una disfunción del ritmo cardíaco causada por una excitabilidad desordenada y de alta frecuencia de las aurículas. La estructura eléctrica y las células que forman las venas pulmonares son las que 
proporcionan la reentrada y la actividad ectópica en la fibrilación auricular. Por lo tanto, este estudio busca señalar datos sobre la epidemiología de la fibrilación auricular y los factores de riesgo relacionados con esta patología. Se trata de una revisión integrativa de la literatura realizada a través de la selección de artículos publicados en revistas indexadas en las bases de datos SciELO y PubMed. En total, se encontraron 329 artículos, de los cuales se seleccionaron 20 literaturas para componer esta revisión. La fibrilación auricular tiene una prevalencia media de 33,5 millones de personas en todo el mundo. Las tasas de prevalencia más altas de fibrilación auricular se encuentran en los países desarrollados, principalmente en América del Norte. La relevancia del sexo en el desarrollo de la enfermedad es discutible, y parece ser similar, excepto en personas mayores de 75 años, cuyo riesgo será mayor en las mujeres por su mayor longevidad. Los factores de riesgo de fibrilación auricular pueden generar cambios auriculares, que pueden ser o no estructurales, que predisponen a los pacientes a desarrollar fibrilación auricular. Algunos de estos factores de riesgo más comunes incluyen diabetes mellitus, alcoholismo, tabaquismo y obesidad. La relevancia de la diferencia de sexo como factor predisponente a la fibrilación auricular aún no es concluyente, pero se ha demostrado que los factores antes mencionados aumentan el riesgo de manifestación de fibrilación auricular.

Palabras-clave: Epidemiología. Factores de riesgo. Fibrilación auricular.

\section{INTRODUÇÃO}

A fibrilação atrial é uma disfunção do ritmo cardíaco por uma excitabilidade de alta frequência e desordenada dos átrios que gera uma irregularidade no padrão excitatório dos ventrículos (Westerman \& Wenger, 2019). Disparos ectópicos focais pelas mangas de miócitos nas veias pulmonares são os precursores da fisiopatologia da fibrilação atrial. A estrutura elétrica e as células que constituem as veias pulmonares são os provedores da reentrada e da atividade ectópica da FA. Anormalidades eletrofisiológicas e estruturais no átrio proporcionam a continuidade da fibrilação ao estabilizar a reentrada podendo chegar à cronicidade do distúrbio. (Staerk et al., 2017).

Segundo Zhang et al. (2021), a fibrilação atrial (FA), que é a arritmia mais comum nos prontos-socorros do País, tem gerado impacto na saúde pública. A prevalência da doença no mundo é estimada em 37,6 milhões de casos e a projeção é um aumento de 60\% até o ano de 2050.

Os fatores de risco de fibrilação atrial (FA) podem gerar alterações histopatológicas e estruturais no átrio (inflamação, fibrose, alterações celulares e moleculares). Essas características incrementam a possibilidade de FA persistente (crônica), bem como podem levar a outros fatores de riscos adicionais que modificam a arquitetura atrial e levam a um desfecho clínico não favorável ao paciente (Staerk et al., 2017).

Os argumentos supracitados associados aos múltiplos fatores de risco que recebem a categoria de modificáveis, (tabagismo, etilismo, hipertensão, diabetes, sedentarismo e obesidade), ou não modificáveis, (idade, sexo, genética e etnia), comprovam a importância de se estudar esses fatores de risco em prol do benefício do paciente e o objetivo de restringir essa patologia a níveis pouco significativos (Zhang et al., 2021).

Portanto, levando-se em consideração que fibrilação atrial é um distúrbio que afeta a maquinaria cardíaca e a qualidade de vida de milhares de pacientes no mundo, o presente estudo justifica-se pelo intento de abordar a epidemiologia e os fatores de risco da fibrilação atrial com o intuito de sintetizar dados referentes à incidência dessa doença, além de elencar à relação desta com exposição à fatores externos (como álcool e tabaco), com grupos especiais (gestantes, atletas e idosos) e com comorbidades pré-existentes (diabetes mellitus). 


\section{MATERIAL E MÉTODOS}

\subsection{Metodologia da pesquisa}

Trata-se de uma revisão integrativa da literatura realizada no mês de novembro de 2021, a qual teve como base a seguinte pergunta norteadora "Como se dá a incidência da FA nos mais diversos grupos e como fatores epigenéticos podem determinar a manifestação dessa doença?”. Após a elaboração da pergunta norteadora, foi realizada a busca de tal tema na literatura.

Sendo assim, por intermédio da seleção de artigos publicados em periódicos indexados nas bases de dados do Scientific Eletronic Library (SciELO) e National Library of Medicine (PubMed), utilizando os seguintes termos descritores conforme orientação dos Descritores em Ciências da Saúde (DeCS): "Epidemiologia", "Fibrilação Atrial" e "Fatores de risco", cruzados pelo operador booleano AND. Desse modo, obteve-se 306 artigos encontrados no PubMed. Já na plataforma Scielo, utilizando-se dos mesmos descritores, obteve-se 23 resultados. Ao todo, somaram-se 329 artigos encontrados; destes, apenas 38 foram selecionadas para leitura.

Os dados foram coletados e análise crítica dos estudos foi devidamente realiza com base nos seguintes critérios bem estabelecidos. Os critérios de inclusão utilizados foram: artigos publicados com texto completo, artigos de revisão sistemática e metanálise, artigos de revisão integrativa de literatura, estudos exclusivos com humanos e referenciados entre 2016-2021. Quanto ao idioma, houve a inclusão apenas de artigos em inglês e em português. Já os critérios de exclusão utilizados foram monografias, cartas ao editor, textos incompletos, trabalhos de conclusão de curso e artigos cujos títulos, resumos não eram coerentes com o foco do presente estudo. Por fim, verificou-se que apenas 18 se enquadraram nos propósitos desta revisão.

\section{RESULTADOS E DISCUSSÃO}

A Fibrilação Atrial (FA) configura-se como a arritmia sustentada mais comum. Recentemente, o estudo Global Burden of Disease demonstrou uma prevalência da FA em 33,5 milhões de pessoas, afetando 2,5\% a 3,2\% da população em todos os países de diversos continentes. As taxas de prevalência mais elevadas de FA são encontradas nos países desenvolvidos, principalmente os da América do Norte. Em contrapartida as taxas mais baixas foram encontradas na Ásia-Pacífico (Dilaveris \& Kennedy, 2016).

De acordo com Aune et al. (2017), os fatores de risco de fibrilação atrial podem gerar alterações atriais, as quais podem ser estruturais ou não, que predispõem pacientes a desenvolver FA. Alguns desses fatores de risco mais comuns incluem diabetes mellitus, alcoolismo, tabagismo e obesidade.

Dos resultados encontrados, as pesquisas mais relevantes que compõem o quesito epidemiológico e dos fatores de risco desse estudo foram sintetizadas na tabela 1 disposta abaixo e serão discutidos a seguir. 
Tabela 1 - Sintetização dos trabalhos utilizados na revisão, publicados no período de 2016 a 2021.

\begin{tabular}{|c|c|c|c|c|c|}
\hline Categorias & Autores (ano) & Título & Revista & Metodologia & $\begin{array}{l}\text { Resultados } \\
\text { Principais }\end{array}$ \\
\hline \multirow{5}{*}{$\begin{array}{l}\text { Estudos } \\
\text { epidemiológicos }\end{array}$} & $\begin{array}{l}\text { Westerman \& } \\
\text { Wenger, } 2019\end{array}$ & $\begin{array}{c}\text { Gender } \\
\text { Differences in } \\
\text { Atrial } \\
\text { Fibrillation: a } \\
\text { review of } \\
\text { epidemiology, } \\
\text { management, } \\
\text { and outcomes. }\end{array}$ & $\begin{array}{c}\text { Current } \\
\text { Cardiology } \\
\text { Reviews }\end{array}$ & Meta-análise & $\begin{array}{l}\text { A relevância do } \\
\text { sexo no } \\
\text { desenvolvimento } \\
\text { da doença é } \\
\text { discutível, exceto } \\
\text { em maiores de } 75 \\
\text { anos, cujo risco } \\
\text { será maior em } \\
\text { mulheres. }\end{array}$ \\
\hline & Xiagdan et. al., 2018 & $\begin{array}{l}\text { Atrial } \\
\text { fibrillation in } \\
\text { athletes and } \\
\text { general } \\
\text { population: A } \\
\text { systematic } \\
\text { review and } \\
\text { meta-analysis }\end{array}$ & Medicine & $\begin{array}{l}\text { Ensaio clínico } \\
\text { randomizado }\end{array}$ & $\begin{array}{c}\mathrm{n}=8.901 \text { pessoas, } \\
\text { dentre as quais, } \\
\text { sendo } 2.308 \text { atletas } \\
\text { e } 6.593 \text { não- } \\
\text { atletas. Os } \\
\text { resultados } \\
\text { evidenciaram que } \\
\text { o risco de FA foi } \\
\text { maior em atletas } \\
\text { especialmente do } \\
\text { sexo masculino. }\end{array}$ \\
\hline & $\begin{array}{l}\text { Chokesuwattanaskul } \\
\text { et al., } 2019\end{array}$ & $\begin{array}{l}\text { Incidence of } \\
\text { atrial } \\
\text { fibrillation in } \\
\text { pregnancy and } \\
\text { clinical } \\
\text { significance: a } \\
\text { meta-analysis }\end{array}$ & $\begin{array}{l}\text { Advances In } \\
\text { Medical Sciences }\end{array}$ & Meta-análise & $\begin{array}{c}\text { Os } 7 \text { estudos de } \\
\text { coorte utilizados } \\
\text { (n=301.638 } \\
\text { gestações) } \\
\text { demonstraram que } \\
\text { a FA possui uma } \\
\text { incidência maior } \\
\text { em gestantes que } \\
\text { têm alguma } \\
\text { doença cardíaca } \\
\text { estrutural (2,2\%) } \\
\text { em relação às que } \\
\text { não possuem } \\
\text { nenhuma } \\
\text { patologia cardíaca } \\
(0,3 \%) .\end{array}$ \\
\hline & \multirow[t]{2}{*}{ Aune et al., 2018} & \multirow[t]{2}{*}{$\begin{array}{c}\text { Diabetes } \\
\text { mellitus, blood } \\
\text { glucose and } \\
\text { the risk of } \\
\text { atrial } \\
\text { fibrillation: A } \\
\text { systematic } \\
\text { review and } \\
\text { meta-analysis } \\
\text { of cohort } \\
\text { studies. }\end{array}$} & $\begin{array}{l}\text { Journal Diabetes } \\
\text { Complications }\end{array}$ & \multirow[t]{2}{*}{ Meta-análise } & $\begin{array}{c}\text { A análise de } 31 \\
\text { estudos } \\
\text { prospectivos ( } \mathrm{n}= \\
10.244 .043 \\
\text { pacientes), sugeriu } \\
\text { que o diabetes } \\
\text { mellitus pode } \\
\text { aumentar o risco } \\
\text { relativo de } \\
\text { fibrilação atrial em } \\
28 \% \text {. }\end{array}$ \\
\hline & & & & & $\begin{array}{c}\text { A análise de } \\
949.496\end{array}$ \\
\hline
\end{tabular}




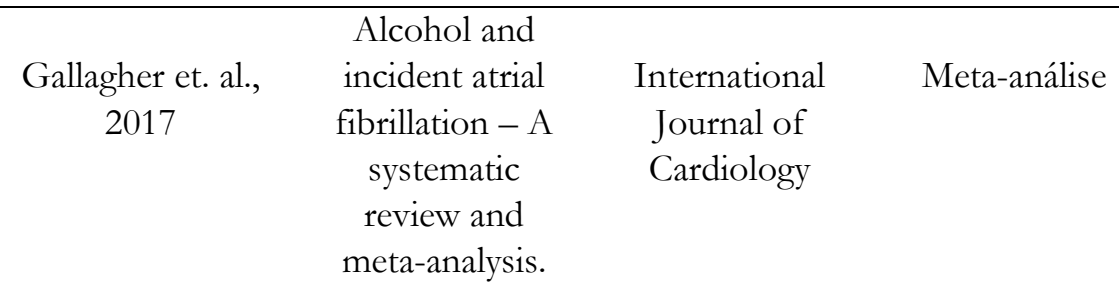

participantes

classificados em

baixo, médio e

alto consumo de

Fatores de risco

meta-analysis.

etanol revelou que

o alto consumo de

álcool aumentou o

risco de $\mathrm{FA}$ em

média de $62 \%$.

\begin{tabular}{|c|c|c|c|c|}
\hline Zhu et al., 2016 & $\begin{array}{l}\text { Association of } \\
\text { smoking with } \\
\text { the risk of } \\
\text { incident atrial } \\
\text { fibrillation: A } \\
\text { meta-analysis } \\
\text { of prospective } \\
\text { studies. }\end{array}$ & $\begin{array}{l}\text { International } \\
\text { Journal of } \\
\text { Cardiology }\end{array}$ & Meta-análise & $\begin{array}{c}\text { O tabagismo } \\
\text { crônico possibilita } \\
\text { um aumento do } \\
\text { risco do } \\
\text { desenvolvimento } \\
\text { de FA em } 23 \% \text { em } \\
\text { comparação com } \\
\text { as pessoas que não } \\
\text { fumam. }\end{array}$ \\
\hline $\begin{array}{c}\text { Asad, Abbas \& } \\
\text { Javed, } 2018\end{array}$ & $\begin{array}{l}\text { Obesity is } \\
\text { associated with } \\
\text { incident atrial } \\
\text { fibrillation } \\
\text { independent of } \\
\text { gender: A } \\
\text { meta-analysis. }\end{array}$ & $\begin{array}{c}\text { Journal of } \\
\text { Cardiovascular } \\
\text { Electrophysiology }\end{array}$ & Meta-análise & $\begin{array}{c}\text { Um análise de } \\
\text { estudos de coorte } \\
\text { que totalizou } \\
587.372 \\
\text { indivíduos, } \\
\text { demonstrou que a } \\
\text { obesidade está } \\
\text { associada a um } \\
\text { risco aumentado } \\
\text { de FA em } 51 \% \text { em } \\
\text { comparação com a } \\
\text { população não } \\
\text { obesa }\end{array}$ \\
\hline
\end{tabular}

Fonte da tabela: Produção própria

De acordo com Westerman \& Wenger (2019), a relevância do sexo no desenvolvimento da doença é discutível, e parece ser semelhante, exceto em maiores de 75 anos, cujo risco será maior em mulheres devido ao aumento da longevidade destas. Porém, a coorte ARIC (Atherosclerosis Risk in Communities) constatou que tanto homens quanto mulheres afro-americanos possuem risco menor de desenvolverem FA.

Em contrapartida, um estudo de coorte do Framingham Heart Study relatou que os homens possuem um risco maior de desenvolver FA em comparação com as mulheres. No entanto, as mulheres com FA têm um risco maior de acidentes vasculares cerebrais e aumento da mortalidade em comparação com os homens (Madan et al., 2019).

De acordo com Xiagdan et al. (2018), existem evidências crescentes que os exercícios de resistência em longo prazo podem promover mudanças na estrutura cardíaca induzindo a FA. Foram feitos 9 ensaios com 8.901 pessoas, dentre as quais, 2.308 eram atletas e 6.593 eram não-atletas. Os resultados evidenciaram que o risco de FA foi significativamente maior em atletas especialmente do sexo masculino e com idade abaixo de 60 anos do que na população em geral. 
Além disso, a incidência de fibrilação atrial entre pacientes com tumores relacionados à supressão dos genes proteína homeobox 3 e gene-4, apresentam mais riscos de desenvolver fibrilação atrial do que a população em geral. Sendo o gene-4 relacionado, principalmente, ao câncer de esôfago (Mao et al., 2018).

A fibrilação atrial constitui uma complicação pouco frequente na gravidez, entretanto, existem dados esparsos sobre o prognóstico, prevalência e o manejo da FA na gestação. A FA geralmente se apresenta como uma arritmia benigna e autolimitada, mas que podem ter consequências hemodinâmicas graves em grávidas que sofrem de insuficiência cardíaca, doença cardíaca congênita ou que seja portadora de outras comorbidades (Katsi et al. 2017).

No que diz respeito à Fibrilação Atrial durante a gestação, foram realizados 7 estudos de coorte, incluindo 301.638 gestações, os quais demonstraram que a FA possui uma incidência maior em gestantes que têm alguma doença cardíaca estrutural $(2,2 \%)$ em relação às que não possuem nenhuma patologia cardíaca $(0,3 \%)$, e está relacionado com o aumento da idade gestacional. Entre as mulheres com FA conhecida, a incidência combinada de FA recorrente na gravidez foi de 39,2\%. Ademais, possui uma incidência estimada de 26,6\% para resultados maternos e fetais insatisfatórios, incluindo pequenos para idade gestacional (PIG), Síndrome do desconforto respiratório, hemorragia intraventricular e óbito (Chokesuwattanaskul et al. 2019).

No que tange aos fatores de rico, uma meta-análise realizada por Aune et al. (2018), a qual envolveu 31 estudos prospectivos, que somam juntos uma análise de 10.244.043 pacientes, sugeriu que o diabetes mellitus pode aumentar o risco relativo de fibrilação atrial em 28\%. Além disso, a condição pré-diabética foi associada a um aumento de $20 \%$ no risco relativo para FA, havendo ainda $12 \%$ de aumento no risco para FA a cada elevação $20 \mathrm{mg} / \mathrm{dL}$ de glicose na glicemia.

A associação entre diabetes e fibrilação atrial refere-se ao fato de que pacientes diabéticos apresentam predisposições a outras condições médicas, as quais também aumentam o risco de fibrilação atrial, tais como: inflamação, hipertensão, doença cardíaca coronária, insuficiência cardíaca, hipertrofia ventricular esquerda e remodelações atriais dos tipos estrutural e elétrica (Staerk et al., 2017).

O diabetes também leva ao aumento de produtos finais de glicosilação, o que pode causar fibrose atrial. Desse modo, observou-se que, em diabéticos, a remodelação atrial estrutural promoveu alterações no diâmetro do átrio esquerdo e no tempo de ativação atrial; enquanto a remodelação atrial elétrica gerou encurtamento do período refratário, bem como atraso na condução interatrial (Moss et al., 2017).

Em relação à ingestão de álcool, a associação entre este e a FA foi referida em uma meta-análise realizada por Gallagher et al. (2017), na qual 9 estudos foram incluídos e totalizaram 949.496 participantes analisados. Nesse estudo, as taxas de álcool ingeridas por dia foram classificadas em baixas (10-12g etanol/dia), intermediárias (20-24g etanol/dia) e altas (30-36g etanol/dia).

As conclusões do estudo supracitado revelaram que os consumos crônicos de níveis moderados e altos de álcool estão associados a níveis crescentes de risco de fibrilação atrial, sendo que o alto consumo de álcool aumentou o risco de FA em média de $62 \%$ para ambos os sexos, enquanto níveis moderados de ingestão de álcool conferiram elevação no risco de FA em homens (48\%), mas não em mulheres (Gallagher et al., 2017) 
Os mecanismos que relacionam o álcool à fibrilação atrial incluem o fato de que o uso crônico do etanol promove prolongamentos nos intervalos entre as ondas elétricas detectadas no eletrocardiograma (Onda P, Complexo QRS e Onda T). Sendo assim, os prolongamentos dos intervalos PR, QRS e QT envolvem um encurtamento do período refratário efetivo e uma maior estimulação simpática, tendo como resultado a liberação de catecolaminas e um aumento nível dos ácidos graxos livres do plasma, os quais são considerados arritmogênicos por natureza, ou seja, aumentam a frequência e o débito cardíaco e predispõem à FA (McManus et al., 2016).

Quanto ao ato de fumar cigarro ativamente e o risco de FA incidente, Zhu et al. (2016) numa metaanálise quantificou a associação e demonstrou um aumento do risco em 23\%. Além disso, o tabagismo atual versus o não atual foi associado a um aumento de 33\% no risco e, nas análises de dose-resposta, fumar 10 cigarros por dia e 10 maços-ano foi associado a aumentos de 14\% e 16\% no risco relativo de FA, respectivamente (Aaune, Schlesinger \& Norat, 2018).

Ademais, é necessário reconhecer os efeitos da exposição ao fumo passivo na FA. Uma análise transversal de 2.816 participantes da coorte de descendentes de Framingham Offspring, na qual 82\% destes foram expostos ao fumo passivo em algum momento da infância, observou um aumento de $18 \%$ no risco de FA na idade adulta, como também, uma maior propensão (17\%) entre os filhos de pais fumantes a fumarem. Evidenciando malefícios do fumo passivo ao risco de FA (Groh et al., 2019).

Os estudos sugerem que existe uma relação biológica subjacente entre fumar e o risco de fibrilação atrial, já que a nicotina altera as concentrações plasmáticas de catecolaminas e a condução do canal iônico. Consequentemente, ocorre o aumento do estresse oxidativo e desencadeia a remodelação atrial, o que potencialmente contribui para o aumento do risco de FA. Isso pode ser ainda mais crítico durante o crescimento e desenvolvimento humano na infância e adolescência (Aaune, Schlesinger \& Norat, 2018).

A obesidade é reconhecida como um dos fatores de risco mais importantes para a FA. De acordo com uma meta-análise de estudos de coorte envolvendo 587.372 indivíduos, observou-se que a obesidade, definida como índice de massa corporal $>30 \mathrm{~kg} / \mathrm{m} 2$, está associada a um risco aumentado de FA (aumento relativo de $51 \%$ ) na população em geral, em comparação com a coorte de não obesos e este efeito foi consistente em homens e mulheres (Asad, Abbas \& Javed, 2018).

No entanto, é importante discernir a composição corporal em termos de peso. Fisicamente, o volume e, principalmente, a qualidade do Tecido Adiposo Epicárdico (EAT), estão amplamente relacionados à doença. Dados prospectivos de longo prazo do Framingham Heart Study, com quase 14 anos de acompanhamento, identificaram a obesidade como um importante fator de risco modificável para FA mediado pela dilatação atrial esquerda, como consequência do acúmulo de EAT (Lavie et al., 2017).

\section{CONSIDERAÇÕES FINAIS}

Quanto à epidemiologia, a fibrilação atrial é a arritmia sustentada mais comum, chegando a afetar um percentual significativo da população em todos os países de diversos continentes. Com relação ao sexo, não foi 
evidenciado ainda se esse fator pode influenciar no desenvolvimento da FA. Ademais, evidenciou-se que atletas e pacientes oncológicos apresentaram maior vulnerabilidade ao desenvolvimento de fibrilação atrial.

Em relação aos fatores de risco, tornou-se claro que a diabetes mellitus, o uso crônico de altas doses de álcool, o fumo e a obesidade estão diretamente atrelados à FA, aumentando de forma considerável o risco dessa manifestação.

Sobre as limitações do estudo, constatou-se a carência de artigos recentes que evidenciassem a epidemiologia da fibrilação atrial. Além disso, não foi analisada a relevância do histórico familiar de fibrilação atrial, que constitui a influência genética aliada aos fatores de risco mencionados.

\section{Agradecimentos}

Em primeira instância, gostaria de agradecer aos demais autores e a nossa querida orientadora Marta Lígia por terem somado suas forças para que esse projeto se concretizasse. Em especial, faz-se imprescindível agradecer ao Núcleo de Estudos em Afecções Cardiopulmonares (NUCAP), projeto de pesquisa da Faculdade Santa Maria (FSM), pois este foi o responsável primordial para nos fornecer os subsídios necessários na construção dessa pesquisa.

\section{Conflitos de interesses}

Os autores declaram que não há conflitos de interesse. Todos os autores estão cientes da submissão do artigo.

\section{Contribuições dos autores}

A autora principal Bianca Caldeira Leite teve participação preponderante nas pesquisas dos aspectos epidemiológicos e, especialmente, no estudo dos fatores de risco envolvidos no desenvolvimento da FA, com destaque para Diabetes Mellitus e Álcool, bem como desenvolveu a metodologia utilizada no presente estudo.

O autor Artur Moreno de Andrade Vasconcelos estruturou e desenvolveu todo o aspecto introdutório acerca da fibrilação atrial sob uma perspectiva epidemiológica associada aos fatores de risco.

A autora Cibele Lorena Fernandes foi responsável pela coleta e interpretação dos dados relacionados ao envolvimento da Obesidade e do tabagismo na manifestação de Fibrilação atrial.

A autora Victória Sampaio Moreira direcionou esforços para análise epidemiológica da fibrilação atrial no âmbito mundial, e da pesquisa dos fatores de risco relacionados à gestação.

A autora Júlia Thaís Cruz teve participação diretamente relacionada à coleta de dados sobre a epidemiologia da fibrilação atrial, sua prevalência de acordo com a idade, sexo e seu desenvolvendo em atletas.

O autor e professor Ubiraídys de Andrade Isidório participou da estruturação do trabalho, auxiliando na análise epidemiológica da Fibrilação Atrial. 
Por fim, a Orientadora Marta Lígia Vieira Melo forneceu todos os subsídios necessários para a boa estruturação da presente pesquisa, sendo presente ao longo de todo o processo de construção do estudo e sanando as dúvidas que surgiram ao longo deste.

\section{REFERÊNCIAS}

Asad, Z.; Abbas, M. \& Javed, I. (2018). Obesity is associated with incident atrial fibrillation independent of gender: A meta-analysis. Journal of Cardiovascular Electrophysiology, May;29(5):725-732. DOI: 1111/jce.13458

Aune, D.; Feng, T.; Schlesinger, S.; Janszky, I.; Norat, T.; Riboli, E. (2018). Diabetes mellitus, blood glucose and the risk of atrial fibrillation: A systematic review and meta-analysis of cohort studies. Journal Diabetes Complications, 32(5):501-511. DOI: 10.1016/j.jdiacomp.2018.02.004

Aune, D.; Schlesinger, S.; Norat, T.; Riboli, E. (2018). Tobacco smoking and the risk of atrial fibrillation: A systematic review and meta-analysis of prospective studies. European Journal of Preventive Cardiology, 25(13):14371451. DOI: $10.1177 / 2047487318780435$

Aune, D.; Sen, A.; ó'Hartaigh, B.; Janszky, I.; Romundstad, P. R.; Tonstad, S., Vatten, J. L. (2017). Resting heart rate and the risk of cardiovascular disease, total cancer, and all-cause mortality - A systematic review and doseresponse meta-analysis of prospective studies. Nutrition, Metabolism and Cardiovascular Diseases, 27(6):504-517. DOI: $10.1016 /$ j.numecd.2017.04.004

Chokesuwattanaskul, R.; Thongprayoon, C.; Bathini, T.; O'Corragain, O. A.; Sharma, K.; Prechawat, S.; Ungprasert, P.; Watthanasuntorn, K.; Pachariyanon, P.; Cheungpasitporn, W. (2019). Incidence of atrial fibrillation in pregnancy and clinical significance: a meta-analysis. Advances In Medical Sciences, Elsevier BV, 64 (2); 415-422. DOI: $10.1016 /$ j.advms.2019.07.003

Dilaveris, P. E. \& Kennedy, H. L. (2016) Silent atrial fibrillation: epidemiology, diagnosis, and clinical impact. Clinical Cardiology, [S.L.], 40(6); 413-418. http://dx.doi.org/10.1002/clc.22667

Gallagher, C.; Hendriks, J. M. L.; Eliott, A. D.; Wong, C. X; Rangnekar, G.; Middeldorp, M. E.; Mahajan, R.; Lau, D. H.; Sanders, P. (2017). Alcohol and incident atrial fibrillation - A systematic review and meta-analysis. International Journal of Cardiology, 246 (2017); 46-52. DOI: 10.1016/j.ijcard.2017.05.133

Groh, C. A.; Vittinghoff, E.; Benjamin, E. J.; Dupuis, J.; Marcus, G. M. (2019). Childhood Tobacco Smoke Exposure and Risk of Atrial Fibrillation in Adulthood. Journal of the American College of Cardiology, 74(13); 16581664. DOI: $10.1016 /$ j.jacc.2019.07.060

Katsi, V.; Georgiopoulos, G.; Marketou, M.; Oikonomou, D.; Parthenakis, F.; Makris, T.; Nihoyannopoulos, P.; Vardas, P.; Tousoulis, D. (2017). Atrial fibrillation in pregnancy: a growing challeng. Current Medical Research Opinion, 33(8): 1497-1504. DOI: 10.1080/03007995.2017.1330257

Lavie, C. J.; Pandey, A.; Lau, D. H.; Alpert, M. A.; Sanders, P. (2017). Obesity and Atrial Fibrillation Prevalence, Pathogenesis and Prognosis: Effects of Weight Loss and Exercise. Journal of the American College of Cardiology. 70 (16), 35. DOI: 10.1016 /j.jacc.2017.09.002

McManus, D. D.; Yin, X.; Gladstone, R.; Vittinghoff, E.; Vasan, R. S.; Larson, M. G.; Benjamin, E. J.; Marcus, G. M. (2016). Alcohol Consumption, Left Atrial Diameter, and Atrial Fibrillation. Journal American Heart Association; 14;5(9):e004060. DOI: 10.1161/JAHA.116.004060

Madan, N.; Itchhaporia, D.; Albert, C. M.; Aggarwal, N. T.; Volgman, A. S. (2019). Atrial Fibrillation and Heart Failure in Women. Heart Failure Clinics. Elsevier BV, [S.L.] 15(1); 55-64. http://dx.doi.org/10.1016/j.hfc.2018.08.006 
Mao, L.; Huang, W.; Zou, P.; Dang, X.; Zeng, X. (2018). The unrecognized role of tumor suppressor genes in atrial fibrillation. Gene, [S.L.], 642; 26-31. Elsevier BV. http://dx.doi.org/10.1016/i.gene.2017.11.015

Moss, A. S.; Dimitropoulos, G.; Connolly, D. L.; Lip, G. Y. H. (2017) Considerations and treatment options for patients with comorbid atrial fibrillation and diabetes mellitus. Expert Opin Pharmacother, 18(11):1101-1114. DOI: 10.1080/14656566.2017.1350647

Staerk, L.; Sherer, J. A.; Ko, D.; Benjamin, E. J.; Helm, R. H. (2017). Atrial Fibrillation: Epidemiology, Pathophysiology, and Clinical Outcomes. Circulationc Research, 28;120(9):1501-1517. DOI: 10.1161/CIRCRESAHA.117.309732

Westerman, S. \& Wenger, N. Gender Differences in Atrial Fibrillation: a review of epidemiology, management, and outcomes. Current Cardiology Reviews, 15(2); 136-144. DOI: 10.2174/1573403X15666181205110624

Wong, C. X.; Ganesan, A. N.; Selvanayagam, J. B. (2017). Epicardial fat and atrial fibrillation: current evidence, potential mechanisms, clinical implications, and future directions. European Heart Journal, 38, 1294-1302. DOI: 10.1093 / eurheartj / ehw045.

Xiagdan, L.; Cui, S.; Xuan, D.; Xuan, C.; Xu, D. (2018). Atrial fibrillation in athletes and general population: A systematic review and meta-analysis. Medicine, 97(49); 13405. DOI: 10.1097/MD.0000000000013405.

Zhang, J.; Johnsen, S. P.; Guo, Y.; Lip, G. Y. H. (2021). Epidemiology of Atrial Fibrillation. Cardiac Electrophysiology Clinics, 13(1); 1-23. DOI: 10.1016/j.ccep.2020.10.010

Zhu, W.; Yuan, P.; Shen, Y.; Wan, R.; Hong, K. (2016). Association of smoking with the risk of incident atrial fibrillation: A meta-analysis of prospective studies. International Journal of Cardiology, 218; 259-266. DOI: 10.1016/j.ijcard.2016.05.013. 